\title{
On iterated forcing for successors of regular cardinals
}

\author{
by
}

Todd Eisworth (Cedar Falls, IA)

\begin{abstract}
We investigate the problem of when $\leq \lambda$-support iterations of $<\lambda$-complete notions of forcing preserve $\lambda^{+}$. We isolate a property-properness over diamonds - that implies $\lambda^{+}$is preserved and show that this property is preserved by $\lambda$-support iterations. Our condition is a relative of that presented by Rosłanowski and Shelah in [2]; it is not clear if the two conditions are equivalent. We close with an application of our technology by presenting a consistency result on uniformizing colorings of ladder systems on $\left\{\delta<\lambda^{+}: \operatorname{cf}(\delta)=\lambda\right\}$ that complements a theorem of Shelah [4].
\end{abstract}

1. Definitions. One of the mysteries of iterated forcing theory is the lack of a good solution to the following "equation" for an uncountable regular cardinal $\lambda$ :

$$
\frac{\text { proper forcing }}{\text { countable support iteration }}=\frac{x}{\lambda \text {-support iteration }} \text {. }
$$

The goal of this paper is to present a generalization of properness to the context of larger cardinals. We make no claim that ours is the "right" generalization; however, the proof that our condition is preserved by $\lambda$-support iteration is close to the proof that properness is preserved by countable support iteration and seems quite natural.

Throughout this paper, we make the following assumptions:

- $\lambda$ is a regular cardinal satisfying $\lambda=\lambda^{<\lambda}$.

- $\mathfrak{D}$ is a normal filter on $\lambda$ "with diamonds", i.e., $\mathfrak{D}$ is closed under diagonal intersections, and for every $S \in \mathfrak{D}^{+}$, there is a sequence $\left\langle A_{\delta}: \delta \in S\right\rangle$ such that for every $A \subseteq \lambda$,

$$
\left\{\delta \in S: A \cap \delta=A_{\delta}\right\} \in \mathfrak{D}^{+} .
$$

- $\chi$ is a regular cardinal that is "large enough".

2000 Mathematics Subject Classification: Primary 03E35.

Key words and phrases: uncountable support iterations, proper forcing.

The author would like to thank the Graduate College of the University of Northern Iowa for a Summer Fellowship in 2002 that provided support while this research was carried out. 
We are going to be looking at when $\lambda^{+}$is preserved by $(\leq) \lambda$-support iterations of $(<) \lambda$-complete notions of forcing. Just as in the case of proper forcing, we will have to look at how our forcing notions interact with elementary submodels.

Definition 1.1. Let $N$ be an elementary submodel of $H(\chi)$. We say that $N$ is relevant if

- $|N|=\lambda$,

- $N^{<\lambda} \subseteq N$,

- $N=\bigcup_{\alpha<\lambda} N_{\alpha}$, where $\left\langle N_{\alpha}: \alpha<\lambda\right\rangle$ is a continuous $\in$-increasing sequence of elementary submodels of $H(\chi)$ such that $\left\langle N_{\beta}: \beta \leq \alpha\right\rangle$ $\in N_{\alpha+1}$ and $\left|N_{\alpha}\right|<\lambda$. (We say that $\left\langle N_{\alpha}: \alpha<\lambda\right\rangle$ is a filtration of $N$.)

The natural attempt at generalizing properness results is a definition along the following lines:

Definition 1.2. A notion of forcing $P$ is said to be $\lambda$-proper if for all sufficiently large regular cardinals $\chi$, there is some $x \in H(\chi)$ such that whenever $M$ is a relevant elementary submodel of $H(\chi)$ with $\{P, x\} \in M$ and $p$ is an element of $M \cap P$, there is a condition $q \leq p$ such that

$$
q \Vdash M\left[\dot{G}_{P}\right] \cap \text { Ord }=M \cap \text { Ord. }
$$

Such a condition $q$ is said to be $(M, P)$-generic.

Some of the qualities of properness generalize in a straightforward fashion to this new context. For example, $\lambda$-proper notions of forcing do not collapse $\lambda^{+}$, and it is easy to prove that both $\lambda^{+}$-closed and $\lambda^{+}$-c.c. notions of forcing are $\lambda$-proper. Unfortunately, $\lambda$-properness is not in general preserved in iterations - work of Shelah and Stanley [5] on generalizing Martin's Axiom to $\aleph_{1}$-complete, $\aleph_{2}$-c.c. notions of forcing made this clear in the early 1980's (Weiss's article [6] contains a nice discussion of this phenomenon.) This paper presents a strengthening of $\lambda$-properness that is preserved by appropriate iterations.

The following assumption is necessary for our arguments to work (although we can weaken it slightly). The most important use is in the proof of Corollary 1.6.

Assumption. All notions of forcing under consideration in this paper are assumed to be $\lambda$-closed.

Definition 1.3. Let $P$ be a notion of forcing, and let $N$ be a relevant elementary submodel of $H(\chi)$.

(1) A set $A \subseteq P$ is $\lambda$-linked if every $A_{0} \in[A]^{<\lambda}$ has a lower bound in $P$. 
(2) An $(N, P)$-diamond over $\mathfrak{D}$ is a sequence $\bar{A}=\left\langle A_{\delta}: \delta \in S\right\rangle$ such that - $S \in \mathfrak{D}^{+}$,

- $A_{\delta}$ is a subset of $N_{\delta} \cap P$ with a lower bound in $P$,

- whenever $A \subseteq N \cap P$ is $\lambda$-linked,

$$
\left\{\delta \in S: N_{\delta} \cap A=A_{\delta}\right\} \in \mathfrak{D}^{+} .
$$

(3) In the context of (2), if $N_{\delta} \cap A=A_{\delta}$ then we say that $\bar{A}$ guesses $A$ at $\delta$.

Our first observation is that $(N, P)$-diamonds are nothing mysteriousthey are just regular diamond sequences that have been cosmetically altered.

Lemma 1.4. Let $N$ be a relevant model with filtration $\left\langle N_{\alpha}: \alpha<\lambda\right\rangle$. Further suppose $\mathfrak{D}$ has diamonds. Then for $S \in \mathfrak{D}^{+}$we can find an $(N, P)$ diamond $\left\langle A_{\delta}: \delta \in S\right\rangle$.

Proof. Let $\left\langle B_{\delta}: \delta \in S\right\rangle$ be a $\mathfrak{D}$-diamond sequence, and let $f: \lambda \rightarrow N \cap P$ be a bijection. Given $\delta \in S$, ask if $f\left[B_{\delta}\right]$ is a $\lambda$-linked subset of $N_{\delta} \cap P$. If so, then we let $A_{\delta}=f\left[B_{\delta}\right]$; if not, then let $A_{\delta}$ be $\left\{\mathbf{1}_{P}\right\}$, where $\mathbf{1}_{P}$ denotes the maximal element of $P$.

Now suppose $A$ is a $\lambda$-linked subset of $N \cap P$. Since $\left\langle B_{\delta}: \delta \in S\right\rangle$ is a diamond sequence, we know that the set of $\delta$ for which $B_{\delta}=f^{-1}(A) \cap \delta$ is in $\mathfrak{D}^{+}$.

There is a closed unbounded set $C$ such that $f\lceil\delta$ is a bijection between $\delta$ and $N_{\delta} \cap P$. If $\delta \in C$ and $B_{\delta}=f^{-1}(A) \cap \delta$, then $A_{\delta}=N_{\delta} \cap A$. Since $C \in \mathfrak{D}$, we see that $\left\langle A_{\delta}: \delta \in S\right\rangle$ is an $(N, P)$-diamond.

Starting with the next lemma, we use without mention the fact that the filter $\mathfrak{D}$ has a natural interpretation in generic extensions of the universein $V[G]$, we let $\mathfrak{D}$ refer to the normal filter generated by $\mathfrak{D} \cap V$.

Lemma 1.5. Let $\left\langle A_{\delta}: \delta \in S\right\rangle$ be an $(N, P)$-diamond, and let $Q$ be a $\lambda$-closed notion of forcing. If $\dot{A}$ is a $Q$-name for a $\lambda$-linked subset of $N \cap P$, then

$$
\Vdash_{Q}\left\{\delta \in S: N_{\delta} \cap \dot{A}=A_{\delta}\right\} \in \mathfrak{D}^{+} .
$$

Proof. If not, then we can find a condition $q$ as well as a $Q$-name $\dot{A}$ and a sequence $\left\langle\dot{C}_{i}: i<\lambda\right\rangle$ of $Q$-names such that

- $\vdash_{Q} \dot{A}$ is $\lambda$-linked,

- $\Vdash_{Q} \dot{C}_{i} \in \mathfrak{D} \cap V$,

- $q \Vdash \delta \in S \cap \triangle_{i<\lambda} \dot{C}_{i} \Rightarrow A_{\delta} \neq N_{\delta} \cap \dot{A}$.

Since $Q$ is $\lambda$-closed, we can find sequences $\left\langle q_{\alpha}: \alpha<\lambda\right\rangle,\left\langle C_{\alpha}: \alpha<\lambda\right\rangle$, and $\left\langle B_{\alpha}: \alpha<\lambda\right\rangle$ such that 
- $\alpha<\beta<\lambda \Rightarrow q_{\beta} \leq q_{\alpha} \leq q$ in $Q$,

- $C_{\alpha} \in \mathfrak{D}$,

- $B_{\alpha} \subseteq N_{\alpha} \cap P$,

- $q_{\alpha} \Vdash \dot{C}_{\alpha}=C_{\alpha}$ and $N_{\alpha} \cap \dot{A}=B_{\alpha}$.

Define $C=\triangle_{\alpha<\lambda} C_{\alpha}$. Since $\mathfrak{D}$ is a normal filter, we know that $C \in \mathfrak{D}$.

Note that the sequence $\left\langle B_{\alpha}: \alpha<\lambda\right\rangle$ increases with $\alpha$. Define

$$
B=\bigcup_{\alpha<\lambda} B_{\alpha}
$$

It is not hard to see that $B$ is $\lambda$-linked (in the ground model), so there is a $\delta \in S \cap C$ such that $N_{\delta} \cap B=A_{\delta}$. This is a contradiction as $q_{\delta}$ is an extension of $q$, yet

$$
q_{\delta} \Vdash \delta \in S \cap \triangle_{i<\lambda} \dot{C}_{i} \text { and } N_{\delta} \cap \dot{A}=B_{\delta}=A_{\delta} .
$$

Corollary 1.6. If $\bar{A}$ is an $(N, P)$-diamond and $G \subseteq P$ is a generic subset of $P$, then

$$
\left\{\delta \in S: N_{\delta} \cap G=A_{\delta}\right\} \in \mathfrak{D}^{+} .
$$

Proof. This follows because $G$ is $\lambda$-directed, hence $\lambda$-linked.

Definition 1.7. A sequence $\bar{R}=\left\langle\left(R_{\delta}, q_{\delta}\right): \delta \in S\right\rangle$ is said to be an $(N, P)$-rule if

- $\left\langle R_{\delta}: \delta \in S\right\rangle$ is an $(N, P)$-diamond,

- $q_{\delta}$ is a lower bound for $R_{\delta}$ in $N \cap P$,

- if $D \in N$ is a dense subset of $P$, then $q_{\delta} \in D$ for all sufficiently large $\delta \in S$.

Definition 1.8. A notion of forcing $P$ is proper over $\mathfrak{D}$-diamonds if (it is $\lambda$-closed and) there is an $x \in H(\chi)$ such that for every relevant model $N$ with $x \in N$, whenever we are given an $(N, P)$-rule $\bar{R}=\left\langle\left(R_{\delta}, q_{\delta}\right): \delta \in S\right\rangle$, for every $p \in N \cap P$ there is $q \leq p$ such that

$q \Vdash$ for some $C \in \mathfrak{D}$, if $\delta \in S \cap C$ and $R_{\delta}=N_{\delta} \cap \dot{G}_{P}$, then $q_{\delta} \in \dot{G}_{P}$. We say that $q$ is $(N, P, \bar{R})$-generic.

In other words, $q$ is $(N, P, \bar{R})$-generic if $q$ forces that in the generic extension, for $\mathfrak{D}$-almost all $\delta \in S$, if $R_{\delta}$ guesses $N_{\delta} \cap G$, then $q_{\delta} \in G$. We say that $q$ forces $N \cap G$ to obey the rule $\bar{R}$. Note as well that the set $C$ appearing in the above definition is not required to be in the ground model; we only require that such a set can be found in the generic extension. This fact is crucial in the arguments we present.

Proposition 1.9. Suppose $N$ is a relevant model containing $P, \bar{R}$ is an $(N, P)$-rule, and $q$ is $(N, P, \bar{R})$-generic. Then $q$ is $(N, P)$-generic, i.e.,

$$
q \Vdash N\left[\dot{G}_{P}\right] \cap \text { Ord }=N \cap \text { Ord. }
$$


In particular, if $P$ is proper over $\mathfrak{D}$-diamonds, then forcing with $P$ preserves the cardinal $\lambda^{+}$.

2. Iterations. We begin with an outline that shows how properness for $\mathfrak{D}$-diamonds is preserved in a simple two-step iteration. Thus, suppose $P$ is proper for $\mathfrak{D}$-diamonds and $\Vdash_{P} \dot{Q}$ is proper for $\mathfrak{D}$-diamonds.

Fix $x \in H(\chi)$ as required in Definition 1.8 for the partial order $P$, and let $\dot{y}$ be a $P$-name for the parameter associated with $\dot{Q}$ in the generic extension. We claim that for every relevant model $N$ containing $\{x, \dot{y}\}$, whenever we are given an $(N, P * \dot{Q})$-rule $\bar{R}$ and $p * \dot{q} \in N \cap P * \dot{Q}$, we can find an extension of $p * \dot{q}$ that forces $\dot{G}_{P * \dot{Q}}$ to obey the rule $\bar{R}$.

Let $\bar{R}=\left\langle\left(R_{\delta}, p_{\delta} * \dot{q}_{\delta}\right): \delta \in S\right\rangle$ be an $(N, P * \dot{Q})$-rule. For $\delta \in S$, define

$$
A_{\delta}:=\left\{p:(\exists \dot{q})\left[p * \dot{q} \in R_{\delta}\right]\right\} .
$$

If we define $\bar{R} \nmid P=\left\{\left(A_{\delta}, p_{\delta}\right): \delta \in S\right\}$, it is straightforward to verify that $\bar{R} \uparrow P$ is an $(N, P)$-rule.

Now let $G$ be a generic subset of $P$ containing an $(N, P, \bar{R}\lceil P)$-generic condition. In the extension $V[G]$, let us define

$$
S_{1}:=\left\{\delta \in S: N_{\delta} \cap G=A_{\delta}\right\} .
$$

We know

$$
V[G] \models S_{1} \in \mathfrak{D}^{+},
$$

and moreover,

$$
V[G] \models(\exists C \in \mathfrak{D})\left[\delta \in S_{1} \cap C \rightarrow p_{\delta} \in G\right] .
$$

In particular, the set

$$
S_{0}:=\left\{\delta \in S: N_{\delta} \cap G=A_{\delta} \text { and } p_{\delta} \in G\right\}
$$

is in $\mathfrak{D}^{+}$.

For $\delta \in S_{0}$, let us define

$$
B_{\delta}=\left\{\dot{b}[G]: a * \dot{b} \in R_{\delta} \text { for some } a \in P\right\} .
$$

Next, we set

$$
\bar{R} / G=\left\{\left(B_{\delta}, \dot{q}_{\delta}[G]\right): \delta \in S_{0}\right\} .
$$

Back in the ground model, we let $\bar{R} / \dot{G}_{P}$ be a $P$-name for the object $\bar{R} / G$ in the generic extension.

Proposition 2.1. If $p$ is $(N, P, \bar{R}\lceil P)$-generic, then

$$
p \Vdash \bar{R} / \dot{G}_{P} \text { is an }\left(N\left[\dot{G}_{P}\right], \dot{Q}\right) \text {-rule. }
$$

Proof. Let $G$ be a generic subset of $P$ that contains $p$. We will prove in detail that $\left\{B_{\delta}: \delta \in S_{0}\right\}$ is an $(N[G], \dot{Q}[G])$-diamond; the rest of the proposition can be verified by similar means. 
Let $E$ be a $\lambda$-linked subset of $N[G] \cap \dot{Q}[G]$. Define $H$ to be the set of all terms $a * \dot{b}$ satisfying

- $a \in N \cap G$,

- $\dot{b}$ is a $P$-name from $N$,

- $\dot{b}[G] \in E$.

Claim 2.2. $V[G]=H$ is a $\lambda$-linked subset of $N \cap P * \dot{Q}$.

Proof. It should be clear that $H$ is a subset of $N \cap(P * \dot{Q})$, so we concentrate on proving that $H$ is $\lambda$-linked. Let $H_{0} \subseteq H$ have cardinality $<\lambda$. Since $P$ is $\lambda$-closed, we know that $H_{0}$ is in the ground model. Furthermore, $N$ is closed under sequences of length $<\lambda$ so we also know that $H_{0} \in N$ and so there is an ordinal $\delta \in S_{0}$ such that $H_{0} \in N_{\delta}$.

Let us define

$$
H_{0}^{P}=\left\{a: a * \dot{b} \in H_{0} \text { for some } \dot{b}\right\}, \quad H_{0}^{Q}=\left\{\dot{b}: a * \dot{b} \in H_{0} \text { for some } a\right\} .
$$

Clearly $H_{0}^{P}$ and $H_{0}^{Q}$ are elements of $N_{\delta}$ as they are definable from $H_{0}$. Our decision to choose $\delta \in S_{0}$ guarantees that $p_{\delta}$ is a lower bound for $N_{\delta} \cap G$. By our definition of $H$, we may conclude that $p_{\delta}$ is a lower bound for $H_{0}^{P}$.

Now in $V[G]$, we know that $E$ is $\lambda$-linked, so that $\left\{\dot{b}[G]: \dot{b} \in H_{0}^{Q}\right\}$ has a lower bound in $Q$. Since $N_{\delta}[G] \prec V[G]$ there must be a $P$-name $\dot{q} \in N_{\delta}$ such that

$$
N_{\delta}[G]=\dot{q}[G] \text { is a lower bound for }\left\{\dot{b}[G]: \dot{b} \in H_{0}^{Q}\right\} .
$$

We will finish upon verifying that $p_{\delta} * \dot{q}$ is a lower bound for $H_{0}$. Let $a * \dot{b} \in H_{0}$ be given. We know immediately that $p_{\delta} \leq a$, so we need only see

$$
p_{\delta} \Vdash \dot{q} \leq \dot{b} \text {. }
$$

This follows because there must exist $r \in N_{\delta} \cap G$ such that $r \Vdash \dot{q} \leq \dot{b}$ and $p_{\delta} \leq r$. Thus $H$ is a $\lambda$-linked subset of $N \cap(P * \dot{Q})$ in $V[G]$.

To finish our proof that $\left\{B_{\delta}: \delta \in S_{0}\right\}$ is an $(N[G], \dot{Q}[G])$-diamond, we take advantage of Lemma 1.5:

$$
V[G] \models S_{2}:=\left\{\delta \in S: N_{\delta} \cap H=R_{\delta}\right\} \in \mathfrak{D}^{+} .
$$

Note that $S_{2}$ is a subset of the set $S_{1}$ from (2.1), and thus

$$
V[G]=S_{0} \cap S_{1} \in \mathfrak{D}^{+} .
$$

By the definition of $H$, for each $\delta \in S_{0} \cap S_{1}$ we have $N_{\delta}[G] \cap E=B_{\delta}$, and we are done.

The previous definitions make the proof of the following lemma quite easy. 
Lemma 2.3. Suppose $\bar{R}=\left\langle\left(R_{\delta}, p_{\delta} * \dot{q}_{\delta}\right): \delta \in S\right\rangle$ is an $(N, P * \dot{Q})$-rule. If $p \in P$ is $(N, P, \bar{R}\lceil P)$-generic, and

$$
p \Vdash \dot{q} \text { is }\left(N\left[G_{P}\right], \dot{Q}, \bar{R} / \dot{G}_{P}\right) \text {-generic, }
$$

then $p * \dot{q}$ is $(N, P, \bar{R})$-generic.

Proof. The proof is a straightforward application of the definitions involved. Let $G^{*}$ be any generic subset of $P * \dot{Q}$ containing $p * \dot{q}$, and let $G \subseteq P$ and $H \subseteq \dot{Q}[G]$ be the canonical factorizations of $G^{*}$ into generic subsets of $P$ and $\dot{Q}[G]$.

In $V\left[G^{*}\right]$, let $E_{1} \in \mathfrak{D}$ exemplify that $p$ is $(N, P, \bar{R} \mid P)$-generic, and let $E_{2} \in \mathfrak{D}$ exemplify that $\dot{q}[G]$ is $(N[G], \dot{Q}[G], \bar{R} / G)$-generic. Suppose now that $\delta \in E_{1} \cap E_{2}$ and $N_{\delta} \cap G^{*}=R_{\delta}$; we must prove that $p_{\delta} * \dot{q}_{\delta} \in G^{*}$.

Let $S_{0}, A_{\delta}$, and $B_{\delta}$ be as in the discussion previous to the statement of our lemma. Since $N_{\delta} \cap G^{*}=R_{\delta}$, we know $N_{\delta} \cap G=A_{\delta}$ and $N_{\delta}[G] \cap H=B_{\delta}$. Since $\delta \in E_{1}$, we must have $p_{\delta} \in G$. Since $\delta \in E_{2}$ (and $\delta \in S_{0}$ ) it follows that $\dot{q}[G] \in H$. Thus $p * \dot{q} \in G^{*}$, as required.

Now what happens with longer iterations? Assume now that $\mathbb{P}=\left\langle P_{i}, \dot{Q}_{i}\right.$ : $i\langle\kappa\rangle$ is a $\lambda$-support iteration of $\lambda$-closed notions of forcing such that

$$
\Vdash_{P_{i}} \dot{Q}_{i} \text { is proper for } \mathfrak{D} \text {-diamonds. }
$$

We will show that $P_{\kappa}$, the limit of $\mathbb{P}$, is proper for $\mathfrak{D}$-diamonds, so in particular forcing with $P_{\kappa}$ preserves $\lambda^{+}$.

Theorem 1 (Preservation Theorem). Let $\left\langle P_{i}, \dot{Q}_{i}: i<\kappa\right\rangle$ be a $\lambda$-support iteration such that

$$
\Vdash_{P_{i}} \dot{Q}_{i} \text { is proper over } \mathfrak{D} \text {-diamonds. }
$$

Then $P_{\kappa}$ is proper over $\mathfrak{D}$-diamonds.

Definition 2.4. Let $N$ be a relevant model with $\mathbb{P} \in N$, and suppose $i<j$ in $N \cap(\kappa+1)$. Let $\bar{A}=\left\langle A_{\delta}: \delta \in S\right\rangle$ be an $\left(N, P_{j}\right)$-diamond; without loss of generality $S$ consists entirely of limit ordinals. Given $\delta \in S$, we define

$$
\begin{aligned}
A_{\delta}\lceil i & =\left\{p\left\lceil i: p \in A_{\delta}\right\},\right. \\
\bar{A}\lceil i & =\left\langle A_{\delta}\lceil i: \delta \in S\rangle .\right.
\end{aligned}
$$

Similarly, if $\bar{R}=\left\langle\left(R_{\delta}, q_{\delta}\right): \delta \in S\right\rangle$ is an $(N, P)$-rule, we define

$$
\bar{R}\left\lceil i=\left\langle\left( R_{\delta}\left\lceil i, q_{\delta}\lceil i): \delta \in S\right\rangle .\right.\right.\right.
$$

Lemma 2.5. Let $N$ be a relevant model containing $\mathbb{P}$, and let $i<j$ in $N \cap(\kappa+1)$. If $\bar{A}$ is an $\left(N, P_{j}\right)$-diamond, then $\bar{A}\left\lceil i\right.$ is an $\left(N, P_{i}\right)$-diamond. If $\bar{R}$ is an $\left(N, P_{j}\right)$-rule, then $\left.\bar{R}\right\rceil i$ is an $\left(N, P_{i}\right)$-rule.

Proof of the Preservation Theorem. We prove by induction on $j \in N \cap$ $\kappa+1$ that whenever we are given objects $i, \dot{p}$, and $r$ such that 
- $i<j$,

- $r \in P_{i}$,

- $\Vdash_{P_{i}} \dot{p} \in P_{\kappa}$,

- $\dot{p} \in N$,

- $r \Vdash \dot{p}\left\lceil i \in \dot{G}_{P_{i}}\right.$,

- $r$ is $\left.\left(N, P_{i}, \bar{R}\right\rceil i\right)$-generic,

we can find a condition $s \in P_{j}$ such that

- $s\lceil i=r$,

- $s$ is $\left(N, P_{j}, \bar{R}\lceil j)\right.$-generic,

- $s \Vdash \dot{p}\left\lceil j \in \dot{G}_{j}\right.$.

Note that our theorem follows from this construction by letting $i=0$ and $j=\kappa$. Also note that $\dot{p}$ is a name for a condition, and not necessarily a condition itself. We need this extra generality in order to make the inductive proof go through.

CAse 1: $j$ is a successor ordinal. Let $j=j_{0}+1$. Since $j_{0}$ must be in $N \cap(\kappa+1)$, we may apply our induction hypothesis to obtain a condition $s_{0} \in P_{j_{0}}$ such that

- $s_{0}\lceil i=r$,

- $s_{0}$ is $\left(N, P_{j_{0}}, \bar{R}\left\lceil j_{0}\right)\right.$-generic,

- $s_{0}$ forces that $\dot{p}\left\lceil j_{0}\right.$ is in $\dot{G}_{j_{0}}$.

At this point, we are essentially in the case where we are doing a twostep iteration - if we view $P_{j}$ as a two-step iteration $P_{j_{0}} * \dot{Q}_{j_{0}}$, then the arguments presented at the beginning of this section show how to extend $s_{0}$ to the required $\left(N, P_{j}, \bar{R}\lceil j)\right.$-generic condition $s$.

CASE 2: $j$ is a limit ordinal of cofinality $<\lambda$.

Lemma 2.6. Suppose $\varepsilon \in N \cap(\kappa+1)$ satisfies $\operatorname{cf}(\varepsilon)<\lambda$, and we are given sequences $\left\langle i_{\alpha}: \alpha<\operatorname{cf}(\varepsilon)\right\rangle$ and $\left\langle r_{\alpha}: \alpha<\operatorname{cf}(\varepsilon)\right\rangle$ such that

- $\left\langle i_{\alpha}: \alpha<\operatorname{cf}(\varepsilon)\right\rangle$ is a strictly increasing sequence of ordinals in $N \cap \varepsilon$,

- $r_{\alpha}$ is $\left(N, P_{i_{\alpha}}, \bar{R}\left\lceil i_{\alpha}\right)\right.$-generic,

- $\alpha<\beta<\kappa \Rightarrow r_{\beta}\left\lceil i_{\alpha}=r_{\alpha}\right.$.

Then the condition $s:=\bigcup_{\alpha<\operatorname{cf}(\varepsilon)} r_{\alpha}$ is $\left(N, P_{\varepsilon}, \bar{R}\lceil\varepsilon)\right.$-generic.

Proof. Clearly $s \in P_{\varepsilon}$ as we are using $\lambda$-support iteration. Let $G$ be any generic subset of $P_{\varepsilon}$ that contains $s$; we will work in the generic extension $V[G]$.

For $\alpha<\operatorname{cf}(\varepsilon)$, let $G_{\alpha}=G \nmid P_{i_{\alpha}}$. Clearly $r_{\alpha} \in G_{\alpha}$ and $G_{\alpha}$ is a generic subset of $P_{i_{\alpha}}$, so there is a set $C_{\alpha} \in \mathfrak{D}$ such that

$$
\delta \in S \cap C_{\alpha} \text { and } N_{\delta} \cap G_{\alpha}=R_{\alpha}\left\lceili _ { \alpha } \Rightarrow q _ { \delta } \left\lceil i_{\alpha} \in G_{\alpha}\right.\right.
$$


Let $C=\bigcap_{\alpha<\operatorname{cf}(\varepsilon)} C_{\alpha} \in \mathfrak{D}$. Given $\delta \in S \cap C$, if $\bar{R}$ guesses $G$ at $\delta$, then (2.5) implies that $q_{\delta}\left\lceil i_{\alpha} \in G_{\alpha}\right.$ for all $\alpha<\operatorname{cf}(\varepsilon)$. Since $G$ is a generic subset of $P_{\varepsilon}$, it follows that $q_{\delta}$ is in $G$, as required.

Now we return to the case where $\operatorname{cf}(j)<\lambda$. Let $\left\langle i_{\alpha}: \alpha<\operatorname{cf}(j)\right\rangle$ be increasing, continuous, and cofinal in $N \cap j$; note that we can achieve continuity because $N$ is closed under sequences of length $<\lambda$. Without loss of generality we assume $i_{0}=i$.

By induction on $\alpha<\operatorname{cf}(j)$, we choose conditions $r_{\alpha} \in P_{i_{\alpha}}$ such that

- $r_{0}=r$,

- $r_{\alpha} \Vdash \dot{p}\left\lceil i_{\alpha} \in \dot{G}_{P_{i_{\alpha}}}\right.$,

- if $\beta<\alpha$ then $r_{\alpha}\left\lceil i_{\beta}=r_{\beta}\right.$,

- if $\alpha$ is a limit ordinal, then $r_{\alpha}=\bigcup_{\beta<\alpha} r_{\beta}$,

- $r_{\alpha}$ is $\left(N, P_{i_{\alpha}}, \bar{R}\left\lceil i_{\alpha}\right)\right.$-generic.

The construction of $\left\langle r_{\alpha}: \alpha<\operatorname{cf}(j)\right\rangle$ is straightforward-at successor stages we apply our induction hypothesis, while at limit stages we invoke Lemma 2.6 to show that the construction continues.

Another application of Lemma 2.6 shows us that the condition $s=$ $\bigcup_{\alpha<\operatorname{cf}(j)} r_{\alpha}$ is $\left(N, P_{j}, \bar{R}\lceil j)\right.$-generic; the other requirements for $s$ are also easily verified.

CASE 3: $\operatorname{cf}(j)=\lambda$. Let $\left\langle i_{\alpha}: \alpha<\lambda\right\rangle$ be increasing, continuous, and cofinal in $N \cap j$ with $i_{0}=i$. Let $\left\langle D_{\alpha}: \alpha<\lambda\right\rangle$ list all dense open subsets of $P_{j}$ that are elements of $N$.

By induction on $\alpha<\lambda$, we will define objects $\dot{p}_{\alpha}$ and $r_{\alpha}$ such that

(1) $r_{0}=r, \dot{p}_{0}=\dot{p} \backslash j$

(2) $r_{\alpha}$ is $\left(N, P_{i_{\alpha}}, \bar{R}\left\lceil i_{\alpha}\right)\right.$-generic,

(3) $r_{\alpha} \backslash i_{\beta}=r_{\beta}$ for $\beta<\alpha$,

(4) $r_{\alpha} \Vdash \dot{p}_{\alpha} \in N \cap P_{j}$ and $\dot{p}_{\alpha}\left\lceil i_{\alpha} \in \dot{G}_{P_{i_{\alpha}}}\right.$,

(5) $r_{\alpha+1} \Vdash \dot{p}_{\alpha+1} \in D_{\alpha}$,

(6) for $\beta<\alpha, r_{\alpha} \Vdash \dot{p}_{\alpha} \leq \dot{p}_{\beta}$,

(7) for $\alpha \in S, r_{\alpha}$ forces the statement

$(\otimes)$ if $q_{\alpha}\left\lceil i_{\alpha} \in \dot{G}_{i_{\alpha}}\right.$ and $q_{\alpha}\left\lceil j\right.$ is a lower bound for $\left\langle\dot{p}_{\beta}: \beta<\alpha\right\rangle$, then $\dot{p}_{\alpha}=q_{\alpha}\lceil j$.

Construction of $\left\langle\dot{p}_{\alpha}: \alpha<\lambda\right\rangle$ and $\left\langle r_{\alpha}: \alpha<\lambda\right\rangle$

InITIAL STAGE. We have already defined $r_{0}$ and $\dot{p}_{0}$.

SuCCESSOR STAGes. Assume now that $\alpha$ is a successor ordinal, say $\alpha=\beta+1$. Our construction will give us objects $r_{\beta}$ and $\dot{p}_{\beta}$ satisfying the appropriate conditions. We apply our induction hypothesis with $i_{\alpha}, i_{\beta}, \dot{p}_{\beta} \backslash i_{\alpha}$, $r_{\beta}$, and $\bar{R}\left\lceil i_{\alpha}\right.$ standing for the objects $j, i, \dot{p}, r$, and $\bar{R}$ appearing there. This gives us an object $r_{\alpha}$ such that 
- $r_{\alpha}$ is $\left(N, P_{i_{\alpha}}, \bar{R}\left\lceil i_{\alpha}\right)\right.$-generic,

- $r_{\alpha}\left\lceil i_{\beta}=r_{\beta}\right.$,

- $r_{\alpha} \Vdash \dot{p}_{\beta} \backslash i_{\alpha} \in \dot{G}_{i_{\alpha}}$.

Now let $G$ be any generic subset of $P_{i_{\alpha}}$ that contains $r_{\alpha}$. We know that $N \cap G$ is $P_{i_{\alpha}}$-generic over $N$ because $r_{\alpha}$ is $\left(N, P_{i_{\alpha}}\right)$-generic. Since $D_{\beta} \in N$, a standard genericity argument tells us that there is a condition $p_{\alpha} \in N[G] \cap$ $P_{j}=N \cap P_{j}$ such that

- $p_{\alpha}\left\lceil i_{\alpha} \in G\right.$,

- $p_{\alpha} \leq \dot{p}_{\beta}[G]$,

- $p_{\alpha} \in D_{\beta}$.

Back in $V$, we let $\dot{p}_{\alpha}$ be a name for this $p_{\alpha}$; it should be clear that $\dot{p}_{\alpha}$ is as required.

Limit STAGES. If $\alpha$ is a limit ordinal, we know

$$
r_{\alpha}=\bigcup_{\beta<\alpha} r_{\beta} .
$$

Since $\operatorname{cf}(\alpha)<\lambda$, Lemma 2.6 implies that $r_{\alpha}$ is $\left(N, P_{i_{\alpha}}, \bar{R}\left\lceil i_{\alpha}\right)\right.$-generic. Also, our inductive assumptions imply that for all $\beta<\alpha$,

$$
r_{\alpha} \Vdash \dot{p}_{\beta}\left\lceil i_{\alpha} \in \dot{G}_{i_{\alpha}}\right. \text {. }
$$

Let $G$ be any generic subset of $P_{i_{\alpha}}$ with $r_{\alpha} \in G$. In the extension $V[G]$, each name $\dot{p}_{\beta}$ is interpreted as a condition $p_{\beta}$ in $N \cap P_{j}$, and we know

- $(\forall \beta<\alpha)\left[p_{\beta}\left\lceil i_{\alpha} \in G\right]\right.$,

- $\left\langle p_{\beta}: \beta<\alpha\right\rangle$ is decreasing.

Now we ask the question: Is it the case that

- $\alpha \in S$,

- $q_{\alpha} \uparrow i_{\alpha} \in G$,

- $q_{\alpha}\left\lceil j\right.$ is a lower bound for $\left\langle p_{\beta}: \beta<\alpha\right\rangle$ in $P_{j}$ ?

If the answer is yes, then we let $p_{\alpha}=q_{\alpha}\lceil j$. If the answer is no, then we let $p_{\alpha} \in N \cap P_{j}$ be a lower bound for $\left\langle p_{\beta}: \beta<\alpha\right\rangle$ in $N \cap P_{j}$ with $p_{\alpha}\left\lceil i_{\alpha} \in G\right.$.

Now back in the ground model, we let $\dot{p}_{\alpha}$ be a name forced by $r_{\alpha}$ to be as above. Note that $\dot{p}_{\alpha}$ is as required in $(\otimes)$, and our construction continues.

Once we have defined $r_{\alpha}$ and $\dot{p}_{\alpha}$ for every $\alpha<\lambda$, we define

$$
s:=\bigcup_{\alpha<\lambda} r_{\alpha} .
$$

Clearly $s\left\lceil i=r\right.$ and $s \Vdash \dot{p}\left\lceil j \in \dot{G}_{j}\right.$, so we need only verify that $s$ is $\left(N, P_{j}, \bar{R}\lceil j)\right.$-generic.

Let $G$ be any generic subset of $P_{j}$ that contains $s$, and step into the model $V[G]$. Each $\dot{p}_{\alpha}$ is interpreted as some $p_{\alpha} \in N \cap P_{j}$ and our construction 
guarantees that the filter generated by $\left\langle p_{\alpha}: \alpha<\lambda\right\rangle$ is generic over $N$ and hence equal to $N \cap G$. This tells us that $s$ is $\left(N, P_{j}\right)$-generic.

For each $\alpha<\lambda$, the condition $r_{\alpha}$ is $\left(N, P_{i_{\alpha}}, \bar{R}\left\lceil i_{\alpha}\right)\right.$-generic so in $V[G]$ we can find a set $C_{\alpha} \in \mathfrak{D}$ that witnesses this, i.e., if $\delta \in C_{\alpha} \cap S$ and $q_{\delta} \backslash i_{\alpha}$ guesses $N_{\delta} \cap G_{i_{\alpha}}$, then $q_{\delta}\left\lceil i_{\alpha} \in G\left\lceil i_{\alpha}\right.\right.$.

Since $\left\langle p_{\alpha}: \alpha<\lambda\right\rangle$ generates $N \cap G$ and $N \cap G$ is generic over $N$, there is a closed unbounded set $E \subseteq \lambda$ such that

$$
\delta \in E \Rightarrow\left\langle p_{\alpha}: \alpha<\delta\right\rangle \text { generates a generic subset of } N_{\delta} \cap P .
$$

Let $C=E \cap \triangle_{\alpha<\lambda} C_{\alpha}$; since $\mathfrak{D}$ is normal, we know that $C \in \mathfrak{D}$.

Claim 2.7. If $\delta \in C \cap S$ and $\bar{R}\left\lceil j\right.$ guesses $N_{\delta} \cap G$, then $q_{\delta}\lceil j \in G$.

Proof. Suppose we are given such a $\delta$. It suffices to show that $q_{\delta}\left\lceil i_{\delta} \in G_{i_{\delta}}\right.$ and $q_{\delta}\left\lceil j\right.$ is a lower bound for $\left\langle p_{\beta}: \beta<\delta\right\rangle$; if this happens, then our construction guarantees $p_{\delta}=q_{\delta}\left\lceil j\right.$ and $p_{\delta} \in G$.

Our definition of $C$ implies that $\delta \in C_{\beta}$ for all $\beta<\delta$. Since $\bar{R}\lceil j$ guesses $N_{\delta} \cap G$, we know that $\left.\bar{R}\right\rceil i_{\alpha}$ guesses $N_{\delta} \cap G_{i_{\alpha}}$ for all $\alpha<\lambda$. Given $\beta<\delta$, we know that $r_{\beta} \in G_{i_{\beta}}$ and $r_{\beta}$ is $\left(N, P_{i_{\beta}}, \bar{R}\left\lceil i_{\beta}\right)\right.$-generic. Putting all this together, we may conclude that for all $\beta<\delta, q_{\delta}\left\lceil i_{\beta} \in G_{i_{\beta}}\right.$, hence $q_{\delta}\left\lceil i_{\delta} \in G_{i_{\delta}}\right.$.

Now why is $q_{\delta}\left\lceil j\right.$ a lower bound for $\left\langle p_{\beta}: \beta<\delta\right\rangle$ ? This follows because $\delta \in C$, i.e. the sequence $\left\langle p_{\beta}: \beta<\delta\right\rangle$ generates $N_{\delta} \cap G$, and we have assumed that $\bar{R}\left\lceil j\right.$ guesses $N_{\delta} \cap G$.

Since $r_{\delta}$ forces $(\otimes)$ to hold, we know that $\dot{p}_{\delta}[G]=q_{\delta}\lceil j$, hence $q_{\delta}\lceil j \in G$.

We have therefore shown that $s$ is $\left(N, P_{j}, \bar{R}\lceil j)\right.$-generic. Since $s\lceil i=r$ and our construction guarantees that $s \Vdash \dot{p}\left\lceil j \in \dot{G}_{P_{j}}\right.$, we see that $s$ is as required.

CASE 4: $\operatorname{cf}(j)>\lambda$. The construction in this case is essentially the same as that of the previous case (in fact, these two cases can easily be handled together by cosmetically altering the argument). Let $k=\sup (N \cap j)$; since $N$ is closed under sequences of length $<\lambda$, it follows that $\operatorname{cf}(k)=\lambda$ and we can fix a continuous increasing sequence $\left\langle i_{\alpha}: \alpha<\lambda\right\rangle$ of elements of $N \cap j$ cofinal in $k$.

The idea now is to mimic the construction given for the case where $\operatorname{cf}(j)=\lambda$. Let $\left\langle D_{\alpha}: \alpha<\lambda\right\rangle$ list all dense open subsets of $P_{j}$ that are elements of $N$. By induction on $\alpha<\lambda$, define objects $\dot{p}_{\alpha}$ and $r_{\alpha}$ satisfying exactly the same requirements as in the previous case-that construction did not require that $j$ was an element of $N \cap \kappa$, only that a sequence along the lines of $\left\langle i_{\alpha}: \alpha<\lambda\right\rangle$ exists. One then checks that the resulting condition $s$ defined as there has all the required properties. 
3. On the $\lambda^{++}$-chain condition. In this section, we give a fairly easy generalization of the fact that $\mathrm{CH}$ implies that the limit of a countable support iteration of proper posets, each of cardinality $\leq \aleph_{1}$, has the $\aleph_{2}$-c.c.; the proof of Proposition 3.1 follows from the proof of Theorem 2.7 of [1] mutatis mutandis. We include a proof of this result for completeness and for the convenience of future citations. We use the standard notation $S_{\lambda^{+}}^{\lambda^{++}}$to denote $\left\{\delta<\lambda^{++}: \operatorname{cf}(\delta)=\lambda^{+}\right\}$.

Proposition 3.1. Assume $\lambda^{<\lambda}=\lambda, 2^{\lambda}=\lambda^{+}$, and let $\mathbb{P}=\left\langle P_{i}, \dot{Q}_{i}\right.$ : $\left.i<\lambda^{++}\right\rangle$be a $\lambda$-support iteration such that

(1) $P_{i}$ is $\lambda$-proper for $i \leq \lambda^{++}$,

(2) $\Vdash_{P_{i}}\left|\dot{Q}_{i}\right| \leq \lambda^{+}$.

Then $P_{\lambda^{++}}$satisfies the $\lambda^{++}$-chain condition.

Proof. Let $\left\{p_{\xi}: \xi<\lambda^{++}\right\}$be given. For each $\xi<\lambda^{++}$, let us fix a model $M_{\xi} \prec H(\chi)$ such that

- $\left\{\mathbb{P}, \xi, p_{\xi}\right\} \in M_{\xi}$,

- $\left|M_{\xi}\right|=\lambda$,

- $M_{\xi}$ is closed under sequences of length $<\lambda$.

By an application of the $\triangle$-system lemma, without loss of generality there is a set $H \subseteq \lambda^{++}$such that

$$
\xi \neq \zeta \Rightarrow M_{\xi} \cap M_{\zeta} \cap \lambda^{++}=H .
$$

For each $\xi<\lambda^{++}$, let $\bar{M}_{\xi}$ be the transitive collapse of $M_{\xi}$. Each $\bar{M}_{\xi}$ is an element of $H\left(\lambda^{+}\right)$and since $\left|H\left(\lambda^{+}\right)\right|=2^{\lambda}=\lambda^{+}$, without loss of generality there is a structure $\bar{M} \in H\left(\lambda^{+}\right)$such that $\bar{M}_{\xi}=\bar{M}$ for all $\xi<\lambda^{++}$. Let $\pi_{\xi}: M_{\xi} \rightarrow \bar{M}$ be the Mostowski isomorphism between $M_{\xi}$ and $\bar{M}$. Since $|\bar{M}|=\lambda$, without loss of generality

$$
\xi \neq \zeta \Rightarrow \pi_{\xi}\left(p_{\xi}\right)=\pi_{\zeta}\left(p_{\zeta}\right) .
$$

Putting all this together, we see that without loss of generality we may assume that for $\xi \neq \zeta$, there is an isomorphism $h_{\xi, \zeta}: M_{\xi} \rightarrow M_{\zeta}$ such that $h_{\xi, \zeta}\left(p_{\xi}\right)=p_{\zeta}$.

Claim 3.2. There is a stationary set $S \subseteq S_{\lambda^{+}}^{\lambda^{++}}$such that $M_{\xi} \cap \xi=H$ for all $\xi \in S$ (where $H$ is the root of our $\triangle$-system).

Proof. For $\xi<\lambda^{++}$, let us define $f(\xi)=\sup \left(M_{\xi} \cap \xi\right)$. Note that $\operatorname{cf}(\xi)=$ $\lambda^{+} \Rightarrow f(\xi)<\xi$, so by Fodor's Lemma there is a stationary set $S_{0} \subseteq S_{\lambda^{+}}^{\lambda^{++}}$ and a $\gamma<\lambda^{++}$such that

$$
\xi \in S_{0} \Rightarrow \gamma=\sup \left(M_{\xi} \cap \xi\right) .
$$


Now $\left|[\gamma]^{\lambda}\right|=|\gamma|^{\lambda} \leq\left(\lambda^{+}\right)^{\lambda}=\lambda^{+}$, so there must be a set $A \subseteq \gamma$ and a stationary $S \subseteq S_{0}$ such that

$$
\xi \in S \Rightarrow A=M_{\xi} \cap \xi .
$$

Note it must be the case that $A \subseteq H$ because $A \subseteq M_{\xi} \cap M_{\zeta} \cap \lambda^{++}$for $\xi \neq \zeta$ in $S$. If we choose $\xi \in S \backslash \sup (H)$, then $H \subseteq M_{\xi} \cap \xi$ and hence $H \subseteq A$. Thus $H=A$, and we have

$$
\xi \in S \Rightarrow M_{\xi} \cap \xi=H
$$

as required.

Now let $C \subseteq \lambda^{++}$be the set of ordinals closed under the function $\xi \mapsto$ $\sup \left(M_{\xi} \cap \lambda^{++}\right)$. The set $I:=S \cap C$ is stationary in $\lambda^{++}$, hence of size $\lambda^{++}$. Furthermore, if $\xi<\zeta$ in $I$, then

- $H=M_{\xi} \cap M_{\zeta} \cap \lambda^{++}$,

- $H \subseteq \min \left(M_{\xi} \cap \lambda^{++} \backslash H\right)$,

- $M_{\xi} \cap \lambda^{++} \subseteq \min \left(M_{\zeta} \cap \lambda^{++} \backslash H\right)$.

In other words, the $\triangle$-system $\left\{M_{\xi} \cap \lambda^{++}: \xi \in I\right\}$ is "not entangled", and $H$ is an initial segment of $M_{\xi} \cap \lambda^{++}$for all $\xi \in I$.

Claim 3.3. $H \cap \lambda^{+}$is an initial segment of $\lambda^{+}$. Furthermore if $\xi \in I$ then $M_{\xi} \cap \lambda^{+}=H \cap \lambda^{+}$.

Proof. The second assertion is obvious. The first assertion follows since we assumed that each $M_{\xi}$ is closed under sequences of length $<\lambda$.

Let us now fix once and for all $\xi<\zeta$ in $I$, and let $h: M_{\xi} \rightarrow M_{\zeta}$ be the isomorphism that carries $p_{\xi}$ to $p_{\zeta}$.

Claim 3.4. Suppose $\theta \in H$. Let $p$ be $\left(M_{\xi}, P_{\theta}\right)$-generic. If $r \in M_{\xi} \cap P_{\theta}$ and $p$ extends $r$, then $p$ also extends $h(r)$.

Proof. The proof is by induction on $\theta \in H$.

CASE 1: $\theta$ is a limit ordinal. Let $r \in M_{\xi} \cap P_{\theta}$ be given. The support of $r$ is a subset of $\theta$ of size at most $\lambda$ that is an element of $M_{\xi}$. Since $\lambda \subseteq M_{\xi}$, we know that $\operatorname{supp}(r) \subseteq M_{\xi} \cap \theta$ and hence $\operatorname{supp}(r) \subseteq H$. This implies that $\operatorname{supp}(r)=\operatorname{supp}(h(r))$ because $h\lceil H$ is the identity.

Thus it suffices to prove for all $\mu \in M_{\xi} \cap \theta$ that $p\lceil\mu$ is an extension $h(r)\lceil\mu=h(r\lceil\mu)$, and this follows from the induction hypothesis.

CASe 2: $\theta=\mu+1$ for some $\mu$. Our induction hypothesis implies that $p\lceil\mu$ extends $h(r)\lceil\mu$, so we need to establish that

$$
p\lceil\mu \Vdash p(\mu) \text { extends } h(r)(\mu) \text {. }
$$

We do this by showing that every extension $t$ of $p\left\lceil\mu\right.$ has an extension $t^{\prime}$ such that

$$
t^{\prime} \Vdash r(\mu)=h(r)(\mu) .
$$


How can we do this? This is where we take advantage of the assumption that $\Vdash_{P_{\mu}}\left|\dot{Q}_{\mu}\right| \leq \lambda^{+}$. Without loss of generality, we can assume that every condition in $P_{\mu}$ forces that the underlying set of $\dot{Q}_{\mu}$ is a subset of $\lambda^{+}$, and hence conditions in $\dot{Q}_{\mu}$ are forced to be ordinals $<\lambda^{+}$. Now we know that $M_{\xi} \cap \lambda^{+}=M_{\zeta} \cap \lambda^{+}=H \cap \lambda^{+}$, and so ordinals in $M_{\xi} \cap \lambda^{+}$are fixed by $h$.

So suppose $t$ is an extension of $p\left\lceil\mu\right.$ in $P_{\mu}$. Since $t$ is $\left(M_{\xi}, P_{\mu}\right)$-generic and $r(\mu)$ is forced to be an ordinal $<\lambda^{+}$, we can find $s \in M_{\xi} \cap P_{\mu}$ and $\alpha \in M_{\xi} \cap \lambda^{+}$such that

- $s$ is compatible with $t$,

- $s \Vdash r(\mu)=\alpha$.

By applying the isomorphism $h$, we see that $h(s) \Vdash h(r)[h(\mu)]=h(\alpha)$. However, $\mu$ and $\alpha$ are fixed by $h$, so in fact we achieve

$$
h(s) \Vdash h(r)(\mu)=\alpha .
$$

Let $t^{\prime}$ be a condition witnessing that $s$ and $t$ are compatible in $P_{\mu}$. Clearly $t^{\prime}$ is $\left(M_{\xi}, P_{\mu}\right)$-generic, so our induction hypothesis (applied to $t^{\prime}$ and $s$ ) implies that $t^{\prime}$ extends $h(s)$ as well. Thus

$$
t^{\prime} \Vdash r(\mu)=h(r)(\mu)=\alpha .
$$

Since $t$ was an arbitrary extension of $p\lceil\mu$, we see

$$
p\lceil\mu \Vdash r(\mu)=h(r)(\mu) .
$$

We know that $p\lceil\mu$ forces $p(\mu)$ to extend $r(\mu)$, and therefore

$$
p\lceil\mu \Vdash p(\mu) \text { extends } h(r)(\mu)
$$

as required.

COROLlary 3.5. If $\xi<\zeta$ in I, then $p_{\xi}$ and $p_{\zeta}$ are compatible.

Proof. Let $\theta=\sup (H)$. It suffices to prove that $p_{\xi}\left\lceil\theta\right.$ and $p_{\zeta}\lceil\theta$ have a common extension $r$ in $P_{\theta}$, as

$$
q:=r \cup p_{\xi} \uparrow\left(M_{\xi} \cap \lambda^{++} \backslash H\right) \cup p_{\zeta} \uparrow\left(M_{\zeta} \cap \lambda^{++} \backslash H\right)
$$

gives a common extension of $p_{\xi}$ and $p_{\zeta}$.

This follows easily from what we just proved - let $r$ be an $\left(M_{\xi}, P_{\theta}\right)$-generic condition stronger than $p_{\xi}\left\lceil\theta\right.$. We know that $\operatorname{supp}\left(p_{\xi}\right) \cap \theta=\operatorname{supp}\left(p_{\zeta}\right) \cap \theta \subseteq H$ and for all $\mu \in H, r\left\lceil\mu\right.$ extends both $p_{\xi}\left\lceil\mu\right.$ and $p_{\zeta}\lceil\mu$.

This finishes the proof that $P_{\lambda^{++}}$has the $\lambda^{++}$-chain condition.

4. An example. Let $S \subseteq S_{\omega_{1}}^{\omega_{2}}:=\left\{\delta<\omega_{2}: \operatorname{cf}(\delta)=\omega_{1}\right\}$ be stationary. Recall that a continuous ladder system on $S$ is a family of functions $\bar{\eta}=$ 
$\left\langle\eta_{\delta}: \delta \in S\right\rangle$ such that $\eta_{\delta}$ is a strictly increasing and continuous from $\omega_{1}$ onto a cofinal subset of $\delta$.

A continuous ladder system $\bar{\eta}$ has the club uniformization property if whenever $\bar{c}=\left\langle c_{\delta}: \delta \in S\right\rangle$ is a family of functions from $\omega_{1}$ to $\{0,1\}$, there is a function $h$ such that for all $\delta \in S$, the set $\left\{i<\omega_{1}: c_{\delta}(i)=h\left(\eta_{\delta}(i)\right)\right\}$ contains a closed unbounded subset of $\omega_{1}$.

Shelah [4] has shown that if the Continuum Hypothesis is true, then no continuous ladder system on (all of) $S_{\omega_{1}}^{\omega_{2}}$ has the club uniformization property. If we are looking at a stationary $S \subseteq S_{\omega_{1}}^{\omega_{2}}$ such that $S_{\omega_{1}}^{\omega_{2}} \backslash S$ is stationary as well, then the techniques of [3] show how to build a model where the Continuum Hypothesis holds and continuous ladder systems on $S$ have the club uniformization property. Our goal is to show that the Continuum Hypothesis is compatible with a certain weak version of the club uniformization property for a continuous ladder system $\bar{\eta}=\left\langle\eta_{\delta}: \delta \in S\right\rangle$ on $S:=S_{\omega_{1}}^{\omega_{2}}$.

TheOREm 2. It is consistent with the Continuum Hypothesis that there exists a stationary set $E_{0} \subseteq \omega_{1}$ and a continuous ladder system $\bar{\eta}=\left\langle\eta_{\delta}\right.$ : $\delta \in S\rangle$ on $S:=S_{\omega_{1}}^{\omega_{2}}$ such that for every family of functions $\bar{c}=\left\langle c_{\delta}: \delta \in S\right\rangle$ mapping $\omega_{1}$ to $\{0,1\}$, there is a function $h: \omega_{2} \rightarrow\{0,1\}$ such that for each $\delta \in S$,

$$
\left\{i \in E_{0}: h\left(\eta_{\delta}(i)\right) \neq c_{\delta}(i)\right\} \text { is non-stationary. }
$$

Said another way, for each $\delta \in S$ there is a closed unbounded $C_{\delta} \subseteq \omega_{1}$ such that

$$
i \in C_{\delta} \cap E_{0} \Rightarrow h\left(\eta_{\delta}(i)\right)=c_{\delta}(i),
$$

i.e., $h$ achieves success at almost every point in $\eta_{\delta}\left[E_{0}\right]$.

Proof. We begin by assuming GCH holds in our ground model, and $E_{0}$ is a stationary and co-stationary subset of $\omega_{1}$ for which $\diamond\left(\omega_{1} \backslash E_{0}\right)$ holds. Let $\mathfrak{D}$ be the filter of closed unbounded subsets of $\omega_{1}$ restricted to the set $\omega_{1} \backslash E_{0}$ (so $\mathfrak{D}$ has diamonds). Let us also fix a continuous ladder system $\bar{\eta}=\left\langle\eta_{\delta}: \delta \in S\right\rangle$.

Suppose $\left\langle c_{\delta}: \delta \in S\right\rangle$ is a family of functions each mapping $\omega_{1}$ to $\{0,1\}$. Our first goal is to define a notion of forcing that will adjoin a function $h$ such that (4.1) is satisfied for all $\delta \in S$.

A condition $p$ is simply an approximation to the desired $h$ of size $\leq \omega_{1}$, i.e., $p \in P$ if $p$ is a function satisfying

- $\operatorname{dom}(p) \in\left[\omega_{2}\right]^{\leq \omega_{1}}$,

- $\operatorname{ran}(p) \subseteq\{0,1\}$,

- for all $\delta \in S$,

$$
\left\{i \in E_{0}: p\left(\eta_{\delta}(i)\right) \neq c_{\delta}(i)\right\} \text { is non-stationary. }
$$


Clearly $P$ is $<\omega_{1}$-closed and for each $\alpha<\omega_{2}$, the set of conditions with $\alpha$ in their domain is dense in $P$. Thus forcing with $P$ adds no new countable sequences to the ground model and adjoins a function from $\omega_{2}$ to $\{0,1\}$.

Claim 4.1. $P$ is proper for $\mathfrak{D}$-diamonds.

Proof. Let $N$ be a relevant model with filtration $\left\langle N_{i}: i<\omega_{1}\right\rangle$ and let $p \in N \cap P$ be arbitrary. Suppose $E_{1} \in \mathfrak{D}^{+}$and let $\bar{R}=\left\langle\left(R_{\delta}, q_{\delta}\right): \delta \in E_{1}\right\rangle$ be an $(N, P)$-rule. Note that we may assume that $E_{0} \cap E_{1}=\emptyset$ because of our definition of $\mathfrak{D}$. We will construct a decreasing sequence $\left\langle p_{\alpha}: \alpha<\omega_{1}\right\rangle$ of conditions in $N \cap P$ in such a way that $q:=\bigcup_{\alpha<\omega_{1}} p_{\alpha}$ is an $(N, P, \bar{R})$-generic extension of $p$.

Let $\gamma=N \cap \omega_{2}$, and for $\alpha<\omega_{1}$ let $\gamma_{\alpha}=N_{\alpha} \cap \omega_{2}$. The sequence $\left\langle\gamma_{\alpha}: \alpha<\omega_{1}\right\rangle$ is strictly increasing, continuous, and cofinal in $\gamma$.

As we build the sequence $\left\langle p_{\alpha}: \alpha<\omega_{1}\right\rangle$, we will also be defining a strictly increasing and continuous sequence of countable ordinals $\left\langle i_{\alpha}: \alpha<\omega_{1}\right\rangle$.

We begin by letting $i_{0}$ be the least $i<\omega_{1}$ such that $p \in N_{i}$, and let $p_{0} \in N \cap P$ be some totally $\left(N_{i_{0}}, P\right)$-generic $\left(^{1}\right)$ extension of $p$.

Given $\left\langle p_{\beta}: \beta \leq \alpha\right\rangle$ and $\left\langle i_{\beta}: \beta \leq \alpha\right\rangle$, we let $i_{\alpha+1}$ be the least ordinal $i$ such that both $\left\langle p_{\beta}: \beta \leq \alpha\right\rangle$ and $\left\langle i_{\beta}: \beta \leq \alpha\right\rangle$ are elements of $N_{i}$. Note that such an $i$ exists because $N^{<\omega_{1}} \subseteq N$. We let $p_{\alpha+1}$ be a totally $\left(N_{i_{\alpha+1}}, P\right)$-generic extension of $p_{\alpha}$ in $N \cap P$.

Now what happens at limit stages of the construction? If $\alpha$ is a limit ordinal, we will be handed $\left\langle p_{\beta}: \beta<\alpha\right\rangle$ and $\left\langle i_{\beta}: \beta<\alpha\right\rangle$. We are committed to the continuity of $\left\langle i_{\alpha}: \alpha<\omega_{1}\right\rangle$, so this means that we are forced to choose

$$
i_{\alpha}=\bigcup_{\beta<\alpha} i_{\beta} .
$$

Let us define

$$
r_{\alpha}=\bigcup_{\beta<\alpha} p_{\beta} .
$$

Since $\alpha$ is a countable ordinal, we know that $r_{\alpha}$ is a condition in $P$, and the relevance of the model $N$ implies that $r_{\alpha} \in N \cap P$. By our construction, we know that $r_{\alpha}$ is totally $\left(N_{i_{\alpha}}, P\right)$-generic; this follows because

$$
N_{i_{\alpha}}=\bigcup_{\beta<\alpha} N_{i_{\beta}} .
$$

Now we ask: Is it the case that

- $i_{\alpha}=\alpha$,

- $\gamma_{\alpha}=\eta_{\gamma}\left(i_{\alpha}\right)$,

- $\alpha \in E_{0} \cup E_{1}$ ?

$\left({ }^{1}\right)$ This means that for every dense open subset of $P$ in $N_{i_{0}}$, there is a condition $r \in N_{i_{0}} \cap P$ such that $p_{0} \leq r$. We can find such conditions because our forcing is countably closed. 
If not, we let $p_{\alpha}=r_{\alpha}$ and the construction continues. If the answer is yes, then we have two cases to consider: the case $\alpha \in E_{0}$ and the case $\alpha \in E_{1}$.

If $\alpha \in E_{0}$, we note first that $\operatorname{dom}\left(r_{\alpha}\right) \subseteq \gamma_{\alpha}$; this is because $p_{\beta} \in N_{\alpha}$ for all $\beta<\alpha$ and $\operatorname{dom}\left(r_{\alpha}\right)=\bigcup_{\beta<\alpha} \operatorname{dom}\left(p_{\beta}\right)$. Thus we may define

$$
p_{\alpha}=r_{\alpha} \cup\left\{\left\langle\gamma_{\alpha}, c_{\gamma}(\alpha)\right\rangle\right\} \text {, }
$$

and conclude that $p_{\alpha} \in N \cap P$.

If $\alpha \in E_{1}$, then we ask if $R_{\alpha}$ is equal to the filter on $N_{\alpha} \cap P$ generated by $\left\langle p_{\beta}: \beta<\alpha\right\rangle$. If yes, then we let $p_{\alpha}=q_{\alpha}$ (note that $q_{\alpha} \leq r_{\alpha}$ if this happens); if not, we let $p_{\alpha}=r_{\alpha}$.

In either case, the condition $p_{\alpha}$ will be in $N \cap P$ and the construction can continue.

Claim 4.2. The sequence $\left\langle p_{\alpha}: \alpha<\omega_{1}\right\rangle$ has a lower bound in $P$.

Proof. Let $q=\bigcup_{\alpha<\omega_{1}} p_{\alpha}$. It is clear that $q$ is a partial function from $\omega_{2}$ to $\{0,1\}$ with domain a set of cardinality $\aleph_{1}$. Since each $p_{\alpha}$ is an element of $N$, we know that $\operatorname{dom}(q) \subseteq \gamma$.

What we need to show is that for every $\delta \in S$, (4.1) holds. If $\delta>\gamma$, then (4.1) holds because $\operatorname{dom}(q) \subseteq \gamma$. If $\delta<\gamma$, we note that $\delta \in N$ (as $N^{<\omega_{1}} \subseteq N$ implies $N \cap \omega_{2}$ is an initial segment of $\omega_{2}$ ), and the set of conditions whose domain includes $\left\{\eta_{\delta}(i): i<\omega_{1}\right\}$ is dense in $P$ and an element of $N$. Thus there is a stage $\alpha$ such that

$$
\left\{\eta_{\delta}(i): i<\omega_{1}\right\} \subseteq \operatorname{dom}\left(p_{\alpha}\right) .
$$

Since $p_{\alpha} \in P$, the definition of $q$ implies (4.1) holds for $\delta$.

The last case to consider is when $\delta=\gamma$. Note that there is a closed unbounded set of $\alpha<\omega_{1}$ for which $i_{\alpha}=\alpha$ and $\eta_{\gamma}(\alpha)=\gamma_{\alpha}$. If $\alpha \in E_{0}$ has these properties, then at stage $\alpha$ we ensured that $q\left(\eta_{\gamma}(\alpha)\right)=c_{\gamma}(\alpha)$. Thus (4.1) holds for $\gamma=\delta$, and we have established that $q$ is a condition in $P$.

Claim 4.3. The condition $q$ is $(N, P, \bar{R})$-generic.

Proof. Again, there is a closed unbounded set of $\alpha$ for which $i_{\alpha}=\alpha$ and $\eta_{\gamma}(\alpha)=\gamma_{\alpha}$. Note that for such an $\alpha$, we automatically achieve that $\left\langle p_{\beta}: \beta<\alpha\right\rangle$ generates an $\left(N_{\alpha}, P\right)$-generic filter $G_{\alpha}$ : this follows because $N_{\alpha}=\bigcup_{\beta<\alpha} N_{i_{\beta}}$. If for such an $\alpha$ it happens that $G_{\alpha}=R_{\alpha}$, then we made sure that $p_{\alpha}=q_{\alpha}$. Since

$$
q \Vdash N \cap \dot{G}_{P} \text { is generated by }\left\langle p_{\alpha}: \alpha<\omega_{1}\right\rangle,
$$

we have ensured that $q$ is $(N, P, \bar{R})$-generic.

This finishes the proof of Claim 4.1.

The actual construction of a model where our weak club uniformization principle holds now follows standard lines - we can use an $\aleph_{1}$-support iteration of length $\aleph_{3}$ (with iterands corresponding to the notion of forcing 
above) to destroy any potential counterexample. The limit of this iteration (i.e., $P_{\omega_{3}}$ ) is countably complete (so $\aleph_{1}$ is preserved), proper for $\mathfrak{D}$-diamonds (so $\aleph_{2}$ is preserved), and $\omega_{3}$-c.c. (so all other cardinals are preserved).

We finish the paper by commenting that there is nothing special about our use of $\omega_{2}$ and $\omega_{1}$ in the previous result; everything generalizes to $\lambda$ and $\lambda^{+}$, where $\lambda$ is regular.

\section{References}

[1] U. Abraham, Proper forcing, chapter in the forthcoming Handbook of Set Theory.

[2] A. Rosłanowski and S. Shelah, Iteration of $\lambda$-complete forcing notions not collap$\operatorname{sing} \lambda^{+}$, Internat. J. Math. Math. Sci. 28 (2001), 63-82.

[3] S. Shelah, Not collapsing cardinals $\leq \kappa$ in $(<\kappa)$-support iterations, Israel J. Math., to appear.

[4] -, Proper and Improper Forcing, Perspect. Math. Logic, Springer, 1998.

[5] S. Shelah and L. Stanley, Generalized Martin's Axiom and Souslin's Hypothesis for higher cardinals, Israel J. Math. 43 (1982), 225-236.

[6] W. Weiss, Versions of Martin's Axiom, in: Handbook of Set-Theoretic Topology, North-Holland, 1984, 827-886.

Department of Mathematics

University of Northern Iowa

Cedar Falls, IA 50614, U.S.A.

E-mail: eisworth@math.uni.edu

Received 10 February 2003;

in revised form 20 October 2003 